\title{
Moldable crystalline $\alpha$-chitin hydrogel with toughness and transparency toward ocular applications
}

Noriyuki Isobe ${ }^{*}$, , Mikiko Tsudome ${ }^{\dagger}$, Ryosuke Kusumi", Masahisa Wada ${ }^{\ddagger}$, Katsuyuki Uematsu" , Satoshi Okada ${ }^{\dagger}$, Shigeru Deguchi ${ }^{\dagger}$

$\dagger$ Research Institute for Marine Resources Utilization, Japan Agency for Marine-Earth Science and Technology (JAMSTEC), 2-15 Natsushima-cho, Yokosuka 237-0061, Japan

$\$$ Division of Forest and Biomaterials Science, Graduate School of Agriculture, Kyoto University, Sakyo-ku, Kyoto 606-8502, Japan

$\S$ Department of Plant and Environmental New Resources, College of Life Sciences, Kyung Hee University, 1732 Deogyeong-daero, Giheung-gu, Yongin-si, Gyeonggi-do 446-701, Republic of Korea

\| Marine Works Japan Ltd, 3-54-1 Oppamahigashi, Yokosuka, Kanagawa, 237-0063, Japan

Corresponding author: Noriyuki Isobe

Corresponding author's email address: isoben@jamstec.go.jp 


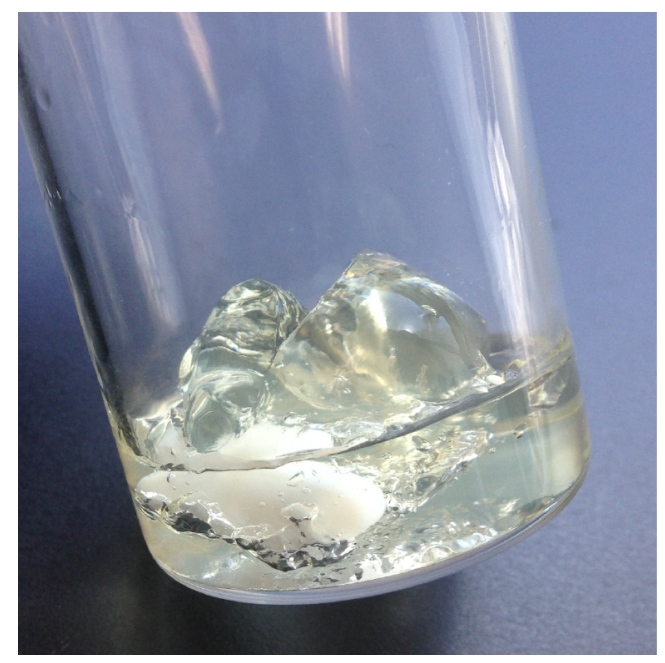

Figure S1. Typical collapsed $N$-acetylated chitosan hydrogel during preparation according to the original protocol. The chitosan solution gelled during mixing with acetylating agent, acetic acid anhydride $\left(\mathrm{Ac}_{2} \mathrm{O}\right)$, before pouring into molds. 


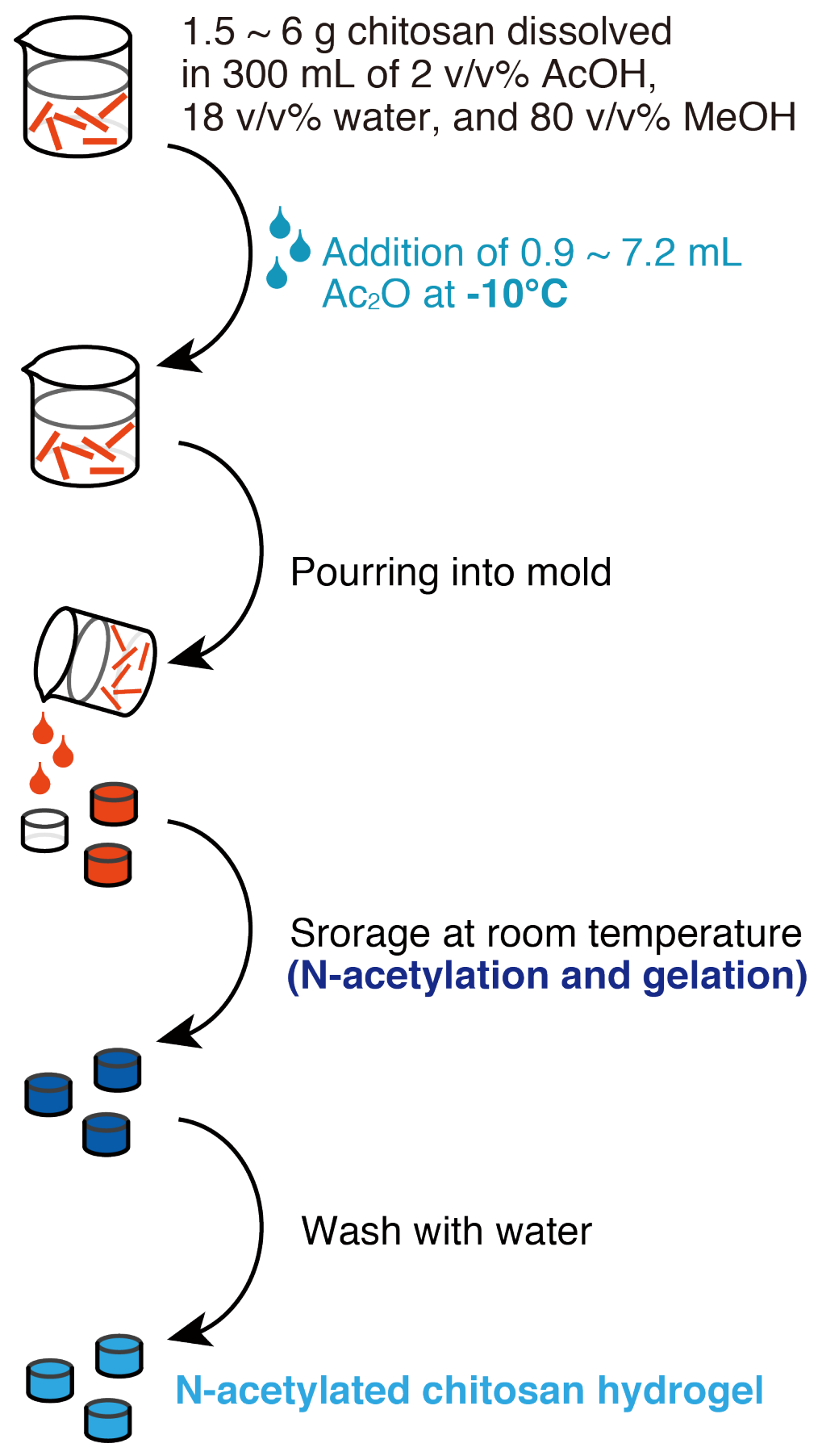

Figure S2. Preparation protocol of $N$-acetylated chitosan hydrogel developed in this study. 


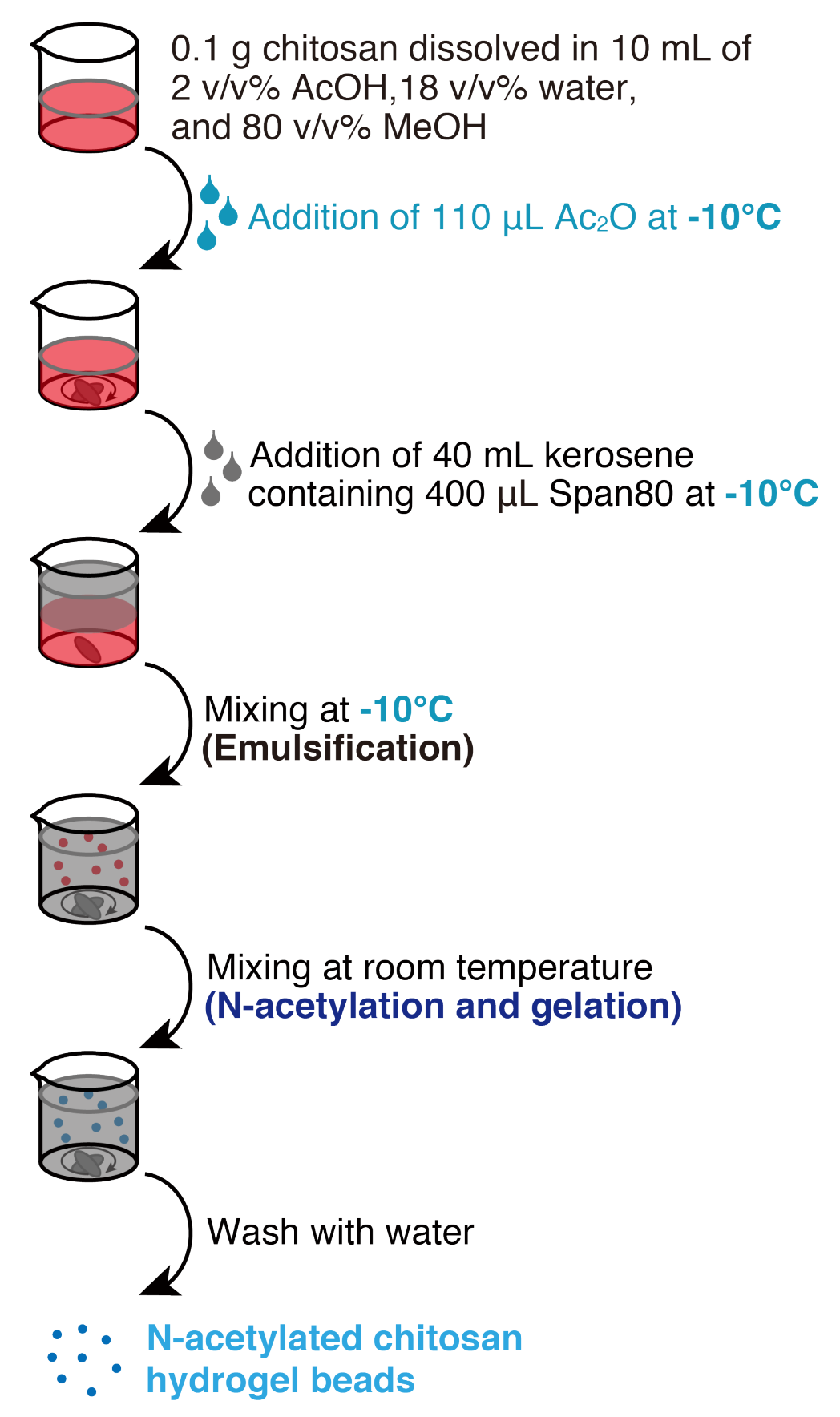

Figure S3. Preparation protocol of $\mathrm{N}$-acetylated chitosan hydrogel beads developed in this study. 


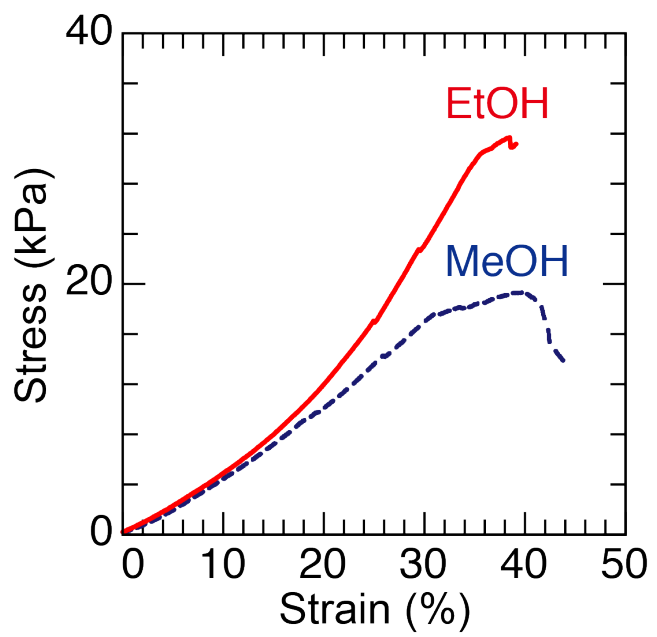

Figure S4. Representative stress-strain (S-S) curves of $N$-acetylated chitosan hydrogels prepared by using methanol (same as "2.3 wt\%" in Fig. 3a) and ethanol.

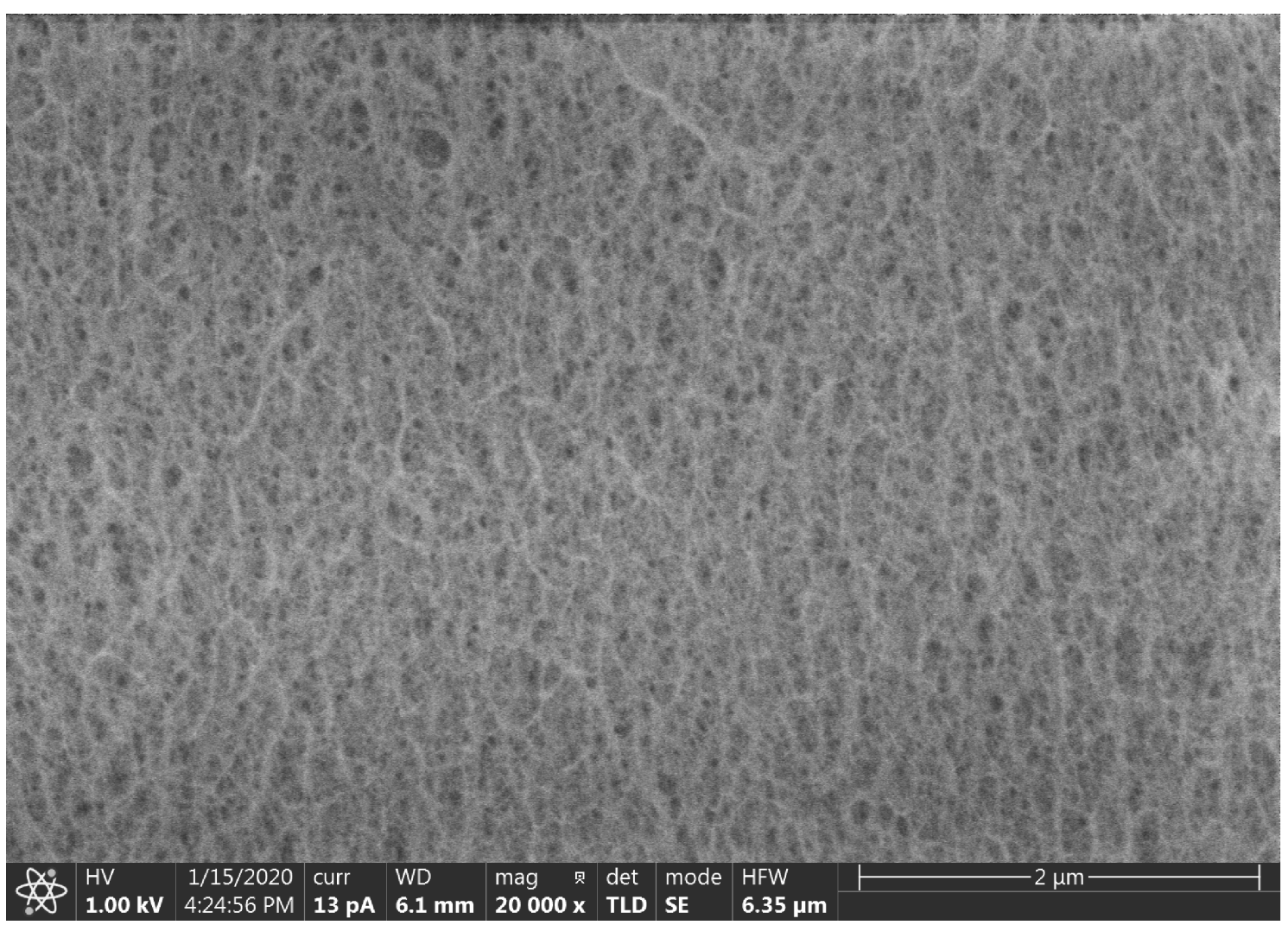

Figure S5. Unmodified electron micrograph of Fig. 5. Cross-sectional cryo-SEM image of $N$-acetylated chitosan gel based on high DP chitosan (DP: 7600). 

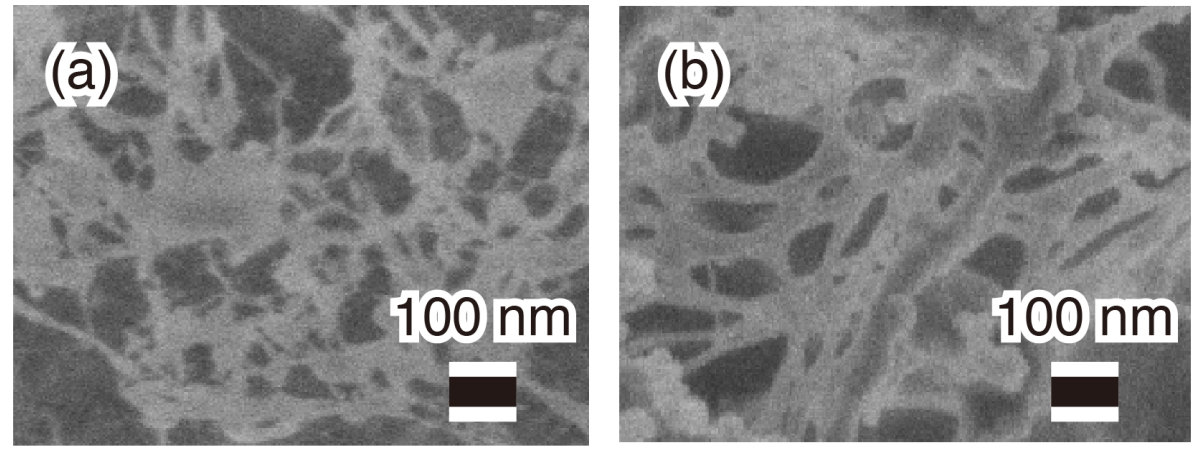

Figure S6. Cross-sectional cryo-SEM image of $N$-acetylated chitosan gel based on low DP chitosan (a) just after gelation and (b) after subsequent washing with water.

Figure S7. Wide angle X-ray diffraction pattern of the $N$-acetylated chitosan hydrogel based on high DP chitosan (DP: 7600) (the same specimen as in Fig. 2f). 


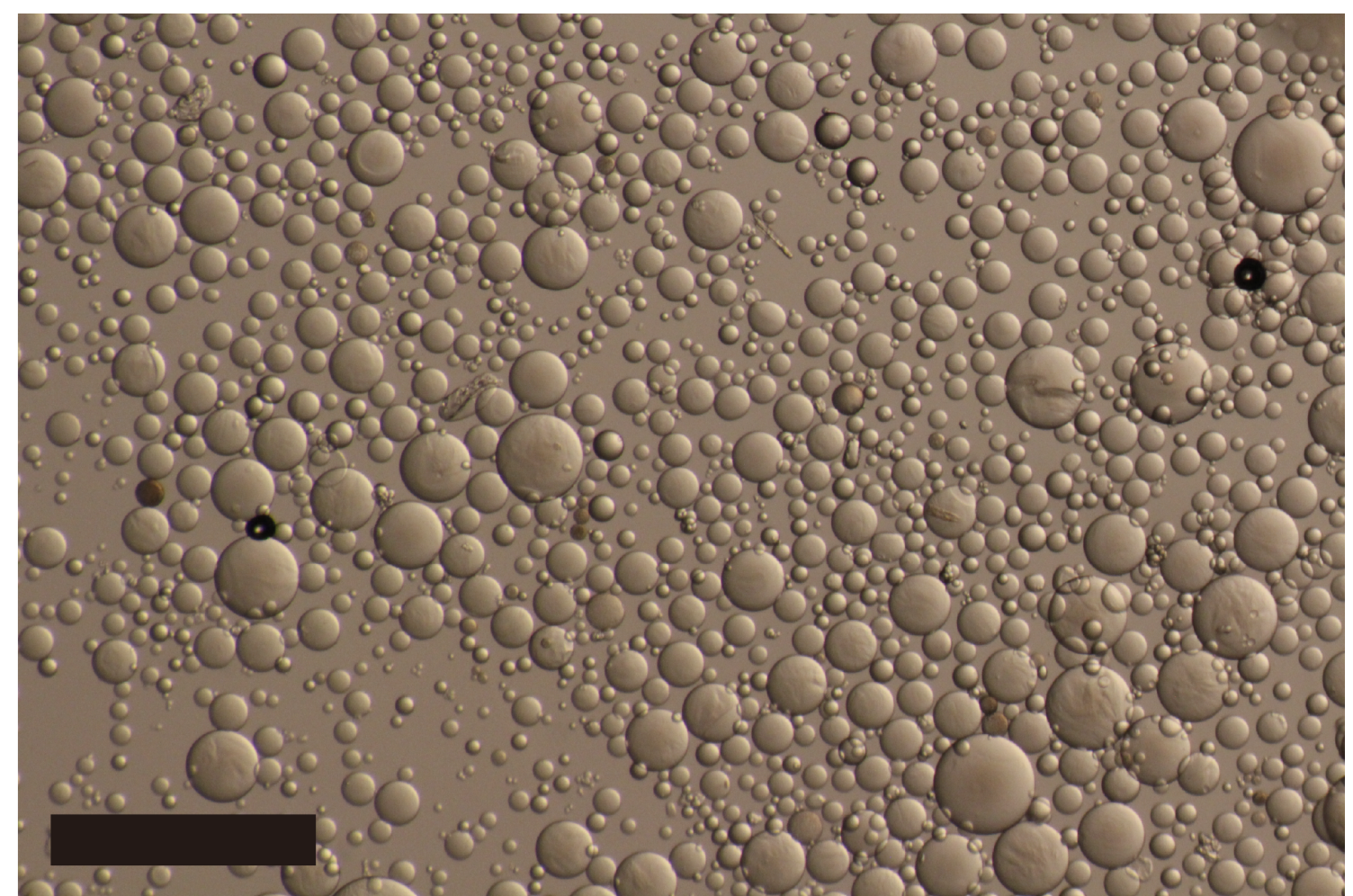

Figure S8. Unmodified optical micrograph of Fig. 8a. Optical micrograph of micro-beads based on $N$-acetylated chitosan hydrogel (DP: 7600). Scale bar represents $1 \mathrm{~mm}$. 